\title{
La levée progressive du tabou des responsabilités socio-professionnelles dans les suicides en lien avec le travail à France Télécom (fin août-octobre 2009)
}

The progressive unveil on the Taboo of the socio-occupational Responsibilities in the Suicides in Connection with the Work at France Telecom (in the End of August-October, 2009)

\section{Alain Rabatel}

\section{(2) OpenEdition} Journals

Édition électronique

URL : http://journals.openedition.org/questionsdecommunication/2045

DOI : 10.4000/questionsdecommunication.2045

ISSN : 2259-8901

Éditeur

Presses universitaires de Lorraine

Édition imprimée

Date de publication : 31 décembre 2011

Pagination : 175-198

ISBN : 978-2-8143-0108-5

ISSN : 1633-5961

Référence électronique

Alain Rabatel, «La levée progressive du tabou des responsabilités socio-professionnelles dans les suicides en lien avec le travail à France Télécom (fin août-octobre 2009) », Questions de communication [En ligne], 20 | 2011, mis en ligne le 01 février 2014, consulté le 01 mai 2019. URL : http:// journals.openedition.org/questionsdecommunication/2045; DOI : 10.4000/ questionsdecommunication.2045 


\title{
$>$ DOSSIER
}

\author{
ALAIN RABATEL \\ Interactions, corpus, apprentissages, représentations \\ Université Lyon 2, ENs-Lyon \\ Université Lyon 1, IUFM \\ alain.rabatel@ens-lyon.fr
}

\section{LA LEVÉE PROGRESSIVE DU TABOU DES RESPONSABILITÉS SOCIO-PROFESSIONNELLES DANS LES SUICIDES EN LIEN AVEC LE TRAVAIL À FRANCE TÉLÉCOM (FIN AOÛT-OCTOBRE 2009)}

\begin{abstract}
Résumé. - Cet article analyse le tabou du traitement médiatique des suicides au travail, à travers l'étude des évolutions de la couverture médiatique des suicides à France Télécom, à la fin de l'été et au début de l'automne 2009, lorsque les suicides se multiplient et prennent des formes spectaculaires. Cette aggravation contraint les dirigeants (patronat et gouvernement) à s'exprimer publiquement et incite les médias à modifier les scénographies antérieures réduites au face-à-face des sources énonciatives patronales et syndicales. Dès lors, la presse manifeste une volonté délibérée d'inscrire chaque suicide dans une série dramatique (à épisodes) et de passer du récit dramatique et spectaculaire à l'explication et à l'interprétation, à travers les modifications du face-à-face antérieur. Cela entraîne l'exploitation plus systématique de l'hyperstructure et le recours aux témoignages des salariés ou des spécialistes de la santé ou du travail. Ces modifications du traitement médiatique (dans la presse quotidienne nationale, régionale ou hebdomadaire) accompagnent, voire accélèrent, l'émergence d'une responsabilité de l'entreprise dans les suicides, et, au-delà, dans la dégradation des relations sociales au travail.
\end{abstract}

Mots clés. - Analyse de discours, politique, hyperstructure, émotion, discours experts, responsabilité. 
$\int_{\text {d }}^{1}$ y a peu d'expérience aussi traumatisante que la confrontation avec le suicide d'un proche - le suicide en deuxième personne pour paraphraser Vladimir Jankélévitch (1977). Mais l'expérience brutale d'un collègue de travail qui se donne la mort - le suicide en troisième personne - est, pour d'autres raisons, tout aussi difficile à gérer, difficile à représenter également, comme le montre la représentation médiatique des suicides qui ont frappé les salariés de France Télécom en 2008 et 2009. L'analyse de discours' à laquelle on procédera en appui sur la linguistique textuelle (Adam, 2005), prolongera une réflexion linguistique sur la responsabilité énonciative mais aussi une réflexion éthique et politique sur la responsabilité citoyenne des journalistes (Rabatel, 2006, 2008, 2009), à travers l'étude de la représentation médiatique des suicides professionnels. En effet, le trait d'union avec lequel nous évoquons la représentation met l'accent sur le fait que cette notion signifie plus qu'une représentation fidèle d'une réalité préexistante. Elle indique une construction nouvelle, une manière de présenter les objets du discours qui joue sur des effets de réel, à des fins argumentatives particulières, en sorte que la re-présentation est la trace de points de vue de l'énonciateur, quand bien même elle prend l'apparence d'une représentation objectivante des choses. Cet ancrage politique, en appui sur la dimension idéologique de la re-présentation, est une manière d'hommage et de fidélité à la puissance critique initiale de l'analyse de discours française (Mazière, 2005 ; Gardin, 1987 (2005a), 1990 (2005b)) - présente encore chez Jacques Guilhaumou $(1998,2006)$ ou dans certains secteurs des sciences humaines, comme le montrent quelques références de ce travail ou encore des publications récentes telles Questions de communication $(16,2009)$ ou Langage et société $($ I 32, 20 I0).

Notre corpus se compose de plus d'une centaine d'articles de presse écrite publiés durant l'année 2009 (PQN,PQR, presse quotidienne gratuite, hebdomadaires, dépêches d'agences, presse spécialisée, sites web)². L'analyse de la matérialité linguistique conduira à faire des choix, car il est impossible de pratiquer des analyses énonciatives de détail sans citer des extraits. Ceux-ci sont néanmoins

\footnotetext{
' Dans un travail non paru à ce jour, Pour une histoire des idées. Les analystes du discours 1968-2002, Recherche FNRS par entretiens filmés, M.-A. Paveau et L. Rosier dénombrent 6 tendances de l'analyse de discours (AD) : AD I ou « Sémantique discursive », initiée par M. Pêcheux; AD2 ou « Discourse Analysis » introduite en France par les textes de Z. Harris et tournée vers une analyse textuelle, formelle et intradiscursive ; AD3 ou « Analyse du discours en interaction (ou Analyse conversationnelle) », autour de C. Kerbrat-Orecchioni ; AD4 ou « Critical discourse analysis » autour de N. Fairclough (GrandeBretagne), R.Wodak (Autriche),T.-A. van Dijk (Espagne/Pays-Bas), D.Vincent (Québec) qui s'intéresse à l'impact du social dans le discours; AD5 ou « Analyse de discours énonciative » avec notamment D. Maingueneau ; AD6 ou « Analyse de discours communicationnelle » travaillant sur le discours médiatique, avec S. Bonnafous et P. Charaudeau. II est certain que des croisements sont toujours possibles, comme le montre ce travail, qui relève de l'AD 5 et 6 , tout en étant proche de préoccupations de l'AD4. Pour une synthèse en ligne, voir l'article de M.-A. Paveau et L. Rosier (2005).

2 Le Monde, Libération, Le Figaro, France Soir, Le Parisien, L'Humanité, 20 Minutes, Le Progrès, Les Dernières Nouvelles d'Alsace, Le Journal du Dimanche, Le Point, L'Express, Le Nouvel Observateur, Télérama, Challenges, L'Expansion, Marianne, Politis, Afr, Santé et Travail, Arrêt sur images, Basta, Médiapart. Nous ne mentionnons ici que les supports cités dans l'article, mais le corpus comprend d'autres titres.
} 
choisis pour leur caractère exemplaire. Le corpus montre une évolution sensible du traitement des informations durant l'été 2009, en passant d'une confrontation globale des responsables de l'entreprise et des syndicats à une représentation qui multiplie les points de vue, ceux d'experts variés, de travailleurs, de responsables politiques, etc. On ne traitera pas ici de la re-présentation des suicides durant la première moitié de l'année 2009 - qui a fait l'objet d'une publication antérieure (Rabatel, 20।0a) - on s'attachera plutôt aux évolutions du traitement médiatique des suicides, à la fin de l'été et au début de l'automne 2009, lorsque les suicides se multiplient, prenant des formes spectaculaires, accusatrices. Cette aggravation contraint d'abord les responsables patronaux et gouvernementaux à bouger publiquement, comme nous le verrons dans une première partie. Elle incite aussi les médias à modifier les scénographies antérieures largement réduites au face-àface des sources énonciatives patronales et syndicales. Dès lors, la presse manifeste une volonté délibérée d'inscrire chaque suicide dans une série dramatique (à épisodes) et de passer du spectaculaire à l'explication et à l'interprétation, à travers les modifications du face-à-face antérieur : ces premiers changements de re-présentation de la mort au travail feront l'objet de la deuxième partie. Dans une troisième partie, nous montrerons que la modification qualitative et quantitative de la re-présentation du suicide passe par l'exploitation plus systématique de l'hyperstructure ${ }^{3}$, avec un recours aux témoignages des salariés ou des spécialistes de la santé ou du travail. Ces modifications du traitement médiatique jouent un rôle important dans l'évolution de la façon dont émerge enfin l'idée d'une responsabilité de l'entreprise dans les suicides, non seulement sur le lieu de travail mais, plus largement, en lien avec le travail, ces issues fatales étant la manifestation la plus dramatique d'un ensemble d'indicateurs de la dégradation des relations sociales au travail. Chemin faisant, le lecteur constatera que les suicides sont rarement montrés, que leur évocation se situe toujours dans un au-delà de la mort qui cherche à en comprendre les motivations. Compte tenu du terreau social du drame, l'explication émerge dans les conflits intenses, inscrivant le suicide dans la chaîne complexe de causes qui ne se limitent pas à l'histoire personnelle des sujets, orientant l'analyse vers l'action, à travers la prise de conscience de la prévention collective des risques.

\section{Les causes externes du tournant dans la couverture médiatique du $20^{\mathrm{e}}$ au $23^{\mathrm{e}}$ suicide}

II est toujours difficile de dater avec précision un tournant dans la couverture d'un événement. Risquons cependant quelques arguments en ce sens, d'abord, de nature externe à la sphère médiatique, ensuite de nature interne. Le tournant prend consistance à la fin de l'été 2009, lorsque toute la presse (presse quotidienne

\footnotetext{
3 L'hyperstructure consiste en un éclatement des longs articles en plusieurs modules textuels, info-
} ou photographiques. La notion est définie plus en détail au début de la troisième section. 
nationale - PQN - presse quotidienne régionale - PQR - gratuits, hebdomadaires, etc.) accorde une place nouvelle, tant quantitativement que qualitativement, à l'évocation des suicides qui se produisent à France Télécom ou qui frappent des salariés chez eux. Si on compare la couverture du $23^{\mathrm{e}}$ suicide avec le traitement de l'information des suicides antérieurs, on constate des évolutions. L'une des explications de ces changements médiatiques est que ce $23^{e}$ suicide émeut plus que les autres, tout en marquant une acmé dans une dégradation récente. La série des suicides et tentatives de suicide s'est accélérée au cours de l'été, souvent dans des circonstances dramatiques depuis le $20^{\mathrm{e}}$ suicide. Ces caractéristiques ont frappé le public et l'ont rendu plus réceptif, comme le confirme la postface de Pierre Morville et Patrick Ackermann au livre d'Yvan du Roy (2009), intitulée «Un été meurtrier », récapitulant la sinistre litanie estivale : Marseille, I 4 juillet ${ }^{4}$, Draguignan, mi juillet, Quimper, 3 I juillet, Besançon, I I août. Lannion, dans la nuit du 29 au 30 août (ce sera le $22^{\mathrm{e}}$ suicide à France Télécom).

Le deuxième élément réside dans le caractère spectaculaire du suicide : en effet, la suicidée, une jeune femme de 32 ans, s'est défenestrée devant ses collègues de travail. Ce geste fait lui-même suite à une tentative de suicide tout aussi spectaculaire, celle d'un employé de Troyes qui s'est poignardé devant une quinzaine de ses collègues et son manager, après qu'on lui eut annoncé la suppression de son poste et sa mutation à une centaine de kilomètres - la direction contestera la distance (voir sites électroniques de 20 Minutes et de L'Expansion le 09/09/09). On est là dans la mort, « dans l'instant mortel » (Jankélévitch, 1997), face aux collègues, et cette dramatisation volontaire est bien évidemment un signal adressé, mettant au cause la disparition des solidarités socioprofessionnelles (Dejours, Bègue, 2009 : 19-20 ; Baudelot, Establet, 2006 : 79-81 ), dont la presse se fait alors l'écho à travers les tribunes offertes à certains sociologues ou psychologues, dont Christophe Dejours (Le Monde, I4/08/09; L'Humanité, 2 1/09/09).

Les déclarations malheureuses de la direction de France Télécom, suite à ces événements, représentent un troisième élément à l'appui du tournant, car ils choquent l'opinion. Les déclarations du PDG, Didier Lombard, sur la mode et la contagion (« La première urgence c'est d'arriver à stopper le phénomène de contagion, en finir avec cette mode des suicides », Le Monde, 17/09/09) sont reprises dans les titres de la presse quotidienne comme dans ceux de la presse hebdomadaire, ce qui leur confère une valeur formulaire (Krieg-Planque, 2009) : ainsi le magazine Télérama (23/09/09) intitule-t-il son article « De la "mode du suicide" », et ouvre par ces mots : « II y aurait une "mode du suicide" » à France Télécom. 23 suicides en dix-huit mois, il est vrai que la tendance est lourde ». Ces

${ }^{4}$ Le cadre qui se suicide à Marseille ( 4/07/09) dénonce dans une lettre sa « surcharge de travail » et un « management par la terreur»(Du Roy, 2009 : 245). Le nouveau PDG de France Télécom reconnaît un an plus tard ce suicide comme accident du travail (voir le site de Libération du |3/07//0). 
propos ont été mal reçus, ce que confirme, par exemple, le titre de Télérama ${ }^{5}$ qui tire les propos maladroits de Didier Lombard en un sens qui n'était sans doute pas celui auquel il pensait, mais qui est néanmoins inférable sans mauvaise foi. L'idée de vague, de contagion n'est pas absurde, elle a de très sérieux garants en la personne d'Émile Durkheim, qui a montré qu'à chaque période de crise, le suicide frappe davantage les individus les plus fragiles. Mais ce qui choque, c'est la volonté de réduire ces suicides à des cas particuliers, alors que, comme le précisait Émile Durkheim (1897), ils renvoient à une dimension sociale incontournable. C'est la raison pour laquelle Jean-Pierre Soubrier, présenté comme psychiatre à l'hôpital américain de Paris (et par ailleurs expert auprès de l'OMS), consulté par L'Expansion, indique avec force qu'« une vague de suicides est révélatrice d'un grave malaise social » (formule durkheimienne qui sert de titre à l'article de Julie de la Brosse dans L'Expansion du 09/09/09).

Un quatrième signe de changement consiste dans le fait que le gouvernement, sous la pression médiatique, est contraint de changer. Xavier Darcos, ministre du Travail,fait plusieurs déclarations qui sont ressenties comme une pression (discrète, mais réelle) sur la direction de France Télécom et missionne son directeur de Cabinet. Toutefois, ces prises de position ne sont pas unanimes. Xavier Bertrand (le 29/09/09 sur France Inter), excuse les dirigeants de l'entreprise qui n'ont rien vu venir, en prétextant que les syndicats n'avaient pas été plus perspicaces, en faisant mine d'oublier la création de l'Observatoire du stress et des mobilités forcées ${ }^{6}$ dès le printemps 2007, ce qui conduit Libération (05/ 10/09) à écrire que «Xavier Bertrand réécrit l'histoire ». Dans le même ordre d'idée, Politis (2009: 8) indique que « Claude Guéant, secrétaire général de l'Elysée, a estimé, comme le directeur des ressources humaines de France Télécom, qu'on ne peut réduire les 23 suicides de salariés "à un problème d'organisation du travail" »».

Le changement touche aussi la direction qui n'est plus dans le déni, voire dans le camouflage explicite d'une vérité qui dérange ${ }^{7}$, et qui accepte enfin de rencontrer

\footnotetext{
${ }^{5}$ Voir également le titre du Progrès dans la section de la troisième partie réservée à la presse quotidienne régionale.

${ }^{6}$ Association co-fondée par les syndicats SUD-PTT, la CFE-CGC et l'UNSA, assistée d'un comité scientifique composé de sociologues, d'ergonomes et de psychothérapeutes, dotée d'un site. Accès : http:// www.observatoiredustressft/org. Cette association a joué un rôle moteur dans la médiatisation des suicides pour faire reconnaître la responsabilité de l'entreprise dans les suicides au travail et, plus largement, dans la dégradation de l'ensemble des relations sociales au travail.

7 Dans Orange stressé (2009: 13-17, 177-|8|), I. du Roy indique qu'il est arrivé à la direction de France Télécom de demander au médecin chef de l'entreprise de réécrire le rapport de ses collègues ou de leur interdire de participer à des réunions des comités d'hygiène et de sécurité, ce qui a conduit le Syndicat national des professionnels de la santé au travail à protester directement auprès de la direction de France Télécom. L'entreprise connaît d'ailleurs une hémorragie de ses médecins. Le sociologue V. de Gaulejac rappelle que lorsqu'un des médecins du travail a écrit à la direction d'BM pour lui signaler l'hyperstress régnant dans l'entreprise, celle-ci a demandé sa radiation au motif qu'il sortait de ses attributions (voir Politis, 17/09/09). Ces faits sont rarement évoqués dans la presse, sans doute pour éviter des procès ou pour ne pas s'attirer les foudres d'un annonceur potentiel.
} 
les syndicats sur cette question le 18 septembre. Mais, là encore, l'avancée n'est pas sans reculs : la direction « récuse tout lien avec son management », parle en effet « de "drames individuels", renvoyant [...] à des problèmes personnels, arguant qu'aucun suicide n'a été qualifié en accident du travail à France Télécom », selon des propos rapportés par SUD-PTT dans L'Humanité (0I/09/09). Aussi les actions envisagées médicalisent-elles et individualisent-elles le problème, avec numéros verts et écoutes psychologiques, tandis qu'elles négligent les rapports de l'individu au groupe ${ }^{8}$.

Ces phénomènes, extérieurs à la sphère médiatique, influent bien évidemment sur sa façon de rendre compte des événements. Voyons à présent les arguments internes à l'appui de la thèse d'un tournant dans le traitement médiatique des suicides en lien avec le travail, avec d'abord la mise en série et l'abandon de la présentation neutralisée des sources, ensuite avec l'hyperstructure, qui justifient qu'on puisse parler d'un nouveau « moment discursif », selon l'expression de Sophie Moirand (2007).

\section{Les évolutions de la re-présentation du suicide avec l'inscription dans le discours primaire de la sériation et l'abandon du retranchement derrière des sources qui se neutralisent}

Soulignons que nous parlons de « la » re-présentation du suicide au singulier, dans la mesure où l'analyse du corpus ne révèle pas de différences significatives de traitement de l'événement dans la presse nationale ou régionale. Compte tenu du poids déterminant des dépêches d'agence, la presse d'information, quelle que soit la sensibilité politique des titres, se borne dans un premier temps à réécrire l'information; certes, la presse d'opinion se distingue par des analyses d'emblée plus complètes et plus critiques, mais comme celle-ci pèse peu, cela n'affecte pas sensiblement les caractéristiques dominantes de la représentation qu'on dégage ici. C'est en ce sens que nous pouvons dire que les évolutions de la re-présentation liées à la sériation et à l'hyperstructure affectent tous les titres, à mesure que la multiplication des drames rend insuffisante la simple opposition des sources syndicales et patronales. Cette évolution passe donc d'une approche psychologisante des suicides - avec une neutralisation des sources (Rabatel, $2010 \mathrm{a}$ ) - à une contextualisation qui dépsychologise la question pour mettre en

\footnotetext{
${ }^{8}$ Sur les limites de ce genre de pratiques, si elles ne s'attaquent pas aux formes d'organisation du travail (voir Santé et travail, 60, 2007, notamment pp. 29-37). La dissolution du social dans le psychologique s'accompagne alors d'un harcèlement de salariés sous « perfusion psychique » : « En mettant l'accent exclusivement sur la relation entre deux individus, l'agresseur et la victime, interprétée à partir de la psychanalyse des perversions, elle [l'approche psychologique compassionnelle et asociale du travail] a frayé la voie à la criminalisation des conduites plus qu'à celle des transformations de l'organisation du travail » (Clot, 2008 : 177).
} 
cause une organisation du travail perverse et délétère, remise en cause rendue possible par le recours aux témoignages directs des salariés et aux analyses des experts qui viennent légitimer la parole syndicale. La re-présentation des suicides se lit d'abord à travers l'inscription de la sériation dans le discours primaire, celui qui est directement pris en charge par le journaliste. La sériation ne se limite pas à l'adjectif numéral ordinal, elle est indiquée par des termes (soulignés dans les citations ci-dessous) qui indiquent la durée, la réitération et qui produisent implicitement des effets accusateurs. Pour illustrer ces procédés, nous nous appuierons sur deux annonces du $25^{\mathrm{e}}$ suicide sur le site de 20 Minutes et du Nouvel Observateur (15/10/09). Le Nouvel Observateur titre sur un « Nouveau suicide $^{9}$ chez France Télécom, en Bretagne », et 20 Minutes « Un 25e salarié de France Télécom s'est suicidé jeudi ». Fait notable, la sériation s'accompagne d'une référence aux sources particulièrement étonnante dans la mesure où l'emploi systématique de selon, pour renvoyer à la source patronale, paraît parfois forcé et produit des effets contreproductifs. Dans 20 minutes, l'article comprend trois sous-titres : «Travail - II avait 48 ans et travaillait à Lannion... », « Le PDG se rend sur place », « Les personnels sont "toujours en danger" ». L'empathie initiale est forte : le suicidé est présenté comme un « ingénieur de 48 ans, en arrêt maladie depuis un mois sur les conseils [sic] de son médecin du travail. Marié et père de famille, il s'est pendu à son domicile, selon la direction » (20 Minutes, 15/10/09).

Dans ce premier paragraphe, la mention de la source est sans doute le signe de ce que la direction est désormais aux avant-postes pour gérer la crise. Cela est confirmé par le deuxième sous-titre, qui focalise sur le PDG - dont on sait qu'il a beaucoup tardé à se déplacer, lui ou d'autres membres de la direction. Le texte précise qu'il « a été "profondément affecté" par ce geste et a décidé de se rendre "immédiatement" sur place, selon un porte parole de la direction » (20 Minutes, 15/10/09).

La deuxième mention de la source (indiquée par « selon ») confirme une stratégie énonciative et argumentative qui consiste à faire, à dire qu'on fait et à le faire savoir. Mais il y a plus. « Selon » est une préposition cadrative annonçant une opinion, des pensées, des paroles. De ce point de vue, son emploi ne fait pas problème dans le deuxième extrait. Dans le premier, son emploi est plus problématique, il s'agit d'un fait, et la préposition qui serait plus conforme serait « d'après » (Schrepfer-André, 2005). Le lecteur comprend que l'information est donnée par le canal de la direction, et c'est bien évidemment ce que le journaliste veut dire. Mais il est fâcheux de présenter un fait comme une opinion. Cette erreur est certainement la trace d'un processus de réécriture de la dépêche d'agence mais elle est autrement significative en indiquant que, dans des situations complexes, conflictuelles, les journalistes se retranchent derrière la mention obstinée de la source des informations, quitte à brouiller la limite entre fait et opinion, afin d'échapper aux prises de responsabilité liées à ce qui est

\footnotetext{
9 Dans toutes les citations, les soulignements sont de notre fait.
} 
dit dans le discours primaire du locuteur quitte aussi à ce que cette réitération obstinée de la source patronale produise des effets sans doute inattendus en fragilisant les témoignages du patronat, puisque des faits sont ramenés à des opinions. Cette difficulté apparaît également en (5) où le fait de se donner la mort dans telles circonstances peut certes être rapporté d'après la direction, mais plus difficilement selon la direction. Mais la gestion des sources énonciative ne se limite pas à cette seule bizarrerie. Dans les articles antérieurs au tournant, les journalistes opposaient assez systématiquement les informations selon la direction et selon les syndicats (Rabatel, 20 IOa). Or, si ici, les informations selon la direction sont maintenues, fût-ce avec les effets incongrus (mais significatifs) qu'on vient de rapporter, en revanche, la source syndicale disparaît (et conséquemment selon aussi), remplacée par deux occurrences de « il s'agit », « il s'agissait », qui posent les circonstances du drame indépendamment de telle source, ce qui dans la mémoire discursive, revient à crédibiliser les informations données systématiquement, avant le tournant, par les sources syndicales : « II s'agit du deuxième salarié se donnant la mort sur le site de Lannion. Un technicien au centre de recherche avait mis fin à ses jours dans la nuit du 29 au 30 août dernier, sans laisser de message expliquant son geste. II s'agissait alors du vingt-deuxième suicide » (20 Minutes, I5/10/09).

Qui plus est, du fait de la sériation, les circonstances sont évoquées selon une focale plus ample, avec l'histoire du site, invitant à dépasser une l'analyse psychologique qui ne prendrait pas en compte la dimension psycho-sociale du phénomène, qui n'est en rien un cas unique. Dans le contexte, le rappel du suicide antérieur, souligne qu'il y a bien un problème d'organisation du travail, ce qui est confirmé par le sous-titre suivant, rapportant les déclarations des syndicats. Celles-ci sont accréditées d'abord par l'exposé des faits directement pris en charge par le discours primaire dans la citation précédente, ensuite par le fait que les déclarations de la direction sont présentées comme une réponse défensive à la situation, puisque les dirigeants « multiplient les mea culpa. Après D. Lombard, qui avait admis vendredi ne pas avoir "pris en compte suffisamment les signaux" de détresse du personnel, c'était au tour du nouveau numéro 2 du groupe, Stéphane Richard, de reconnaître que l'entreprise était "peut-être allée trop loin" dans les mécanismes de contrôle des salariés » (20 Minutes, I5/10/09).

La nature des verbes de parole (admis, reconnaître) ou la qualification de ces derniers par le journaliste (mea culpa), les modalisations (suffisamment, peutêtre) indiquent un malaise de la direction qui est à la fois défensive et qui, malgré tout, cherche à contre-attaquer. Bref, l'orientation de l'article est ainsi faite que tout converge vers l'accréditation de la parole des syndicats, insistant sur la réalité du stress, qui doit faire l'objet des négociations du 20 octobre. Cette orientation est d'ailleurs annoncée d'emblée par la photo qui chapeaute l'article, montrant les grilles d'un autre centre de France Télécom frappé par un suicide. Or, ces grilles (qui évoquent l'univers pénitentiaire) portent une affichette (« SUICIDES + TENTATIVES DE SUICIDES = SUPPRESSIONS D'EMPLOI »), comme si, du point de vue cynique 
de la direction, la vague de suicides s'inscrivait dans sa stratégie de dégraissage ou de départ volontaires. L'article du Nouvel Observateur, plus court, reprend le même canevas pour l'annonce du suicide - confirmant l'importance du rewriting des dépêches d'agence - et présente des caractéristiques identiques : « Un salarié de France Télécom s'est suicidé à son domicile à Lannion (Côte d'Armor), a confirmé la direction du groupe. [...] L'homme, âgé de 48 ans qui travaillait dans le centre de recherche et de développement du groupe à Lannion, s'est donné la mort à son domicile jeudi matin selon la direction. Elle précise qu'il était en arrêt maladie depuis un mois » (Le Nouvel Observateur, I5/10/09).

Vient ensuite un paragraphe évoquant le déplacement de Didier Lombard « selon la direction », sans mentionner ses paroles. Le paragraphe suivant élargit l'angle d'analyse : «Ce nouveau suicide porte à 25 le nombre de suicides dans le groupe en 19 mois selon les syndicats $\gg$. Le $5^{e}$ paragraphe évoque une récente tentative de suicide à Marseille, puis les deux derniers, les plus longs de l'article, sont consacrés aux syndicats qui évoquent les négociations sur le stress du 20 octobre et mettent en cause l'ancien $n^{\circ} 2$, Louis-Pierre Wenes « accusé de méthodes de management jugées brutales ». L'article accorde une importance croissante aux circonstances du drame et à son explication, en tentant de faire entendre que la répétition des drames est significative d'un problème plus vaste que ce qu'en dit la direction. On est certes dans une communication implicite : l'évocation du remplacement de Louis-Pierre Wenes par Stéphane Richard, appelé dès sa nomination à remplacer Didier Lombard, suggère que ces remplacements au plus haut niveau sont le signe des responsabilités du management dans la dégradation des conditions de travail, car l'hypothèse d'un bouc émissaire n'est pas présente. Le rapport entre suicide et conditions de travail est présenté comme un fait établi, dans chacun des articles, en lui-même, et bien sûr, à travers la réitération dramatique de la série. L'inscription des faits dans une série transforme le fait particulier en un événement, inséré dans une trame causale/explicative. Le suicide n'est plus un cas dramatique particulier évoqué de façon émotionnelle sur la scène médiatique et émotionnelle, il devient un signe dans une série, et celle-ci s'inscrit dans une histoire globale qui a un sens, celui des ravages d'un management libéral (voir Gardin, 1987, 2005a : 232). Comme le souligne Alain Badiou (1988: 202), l'événement est un, par sa globalité, et multiple, par la diversité des faits qui le composent. Toutefois, cette globalité est difficile à émerger et à dégager, car la situation de France Télécom est complexe et relativement inédite, pour une presse qui traite peu des questions sociales, et qui se sent mieux armée pour traiter des faits divers ou des affaires. Au demeurant, tous les spécialistes qui ont travaillé sur la notion d'événement ont souligné que ceux qui sont aisément repérables et analysables sont ceux qui se répètent et s'inscrivent dans une classe, ceux dont on peut prévoir la réitération et le dénouement (Ricœur, 1983 : 48 ; Arquembourg, 2003 : 40). C'est donc progressivement - sauf pour les militants et les journalistes engagés qui ont à leur disposition des grilles d'analyse donnant d'emblée du sens à ces suicides à répétition - que la représentation des suicides à France Télécom ne se limite pas à une simple narration d'un fait, fût-il inscrit dans une série ; 
la trame narrative et la visée informative se doublent de visées explicative et argumentative, assorties d'infléchissements notables de l'explication à mesure que les suicides se multiplient. Mais ces visées explicatives et argumentatives ne se déploient pleinement que dans le cadre des hyperstructures, qui permettent l'émergence d'une dimension praxique, politique, particulièrement importante.

\section{La montée en puissance du traitement analytique des suicides dans des hyperstructures}

Dans le traitement des suicides, le deuxième signe d'une évolution tient à la généralisation de l'emploi d'hyperstructures. Ce terme - forgé par Ernest U. Grosse et Ernest Seibold (1994:54) en référence aux usages du net qui permettent de naviguer de lien en lien dans les hypertextes ${ }^{10}$ - renvoie aux évolutions en cours dans la presse écrite comme sur le net (de Broucker, 1995 : 75 ; Mouriquand, 1997 : 109 ; Adam, Lugrin 2000, 2006 ; Lugrin, 2000). Formellement, l'hyperstructure consiste en un éclatement des longs articles hétérogènes au plan des types de texte en de petits modules, plus homogènes au plan du type de texte, indépendants les uns des autres, et donc, souvent encadrés. Cet essaimage, qui donne aux hyperstructures une structure réticulaire (Herrenschmidt, 2007), se combine avec une augmentation de la part visuelle (photographies et infographies), afin de faciliter la compréhension et de permettre des parcours de lecture plus libres. En général, les hyperstructures se déploient sur une page ou une double page, mais elles peuvent aussi occuper moins d'une page. On les retrouve dans la PQN comme dans la PQR, avec des interviews de spécialistes du travail, du suicide, de syndicalistes, d'hommes politiques - la PQR allant rarement jusqu'à des hyperstructures occupant une double page entière. L'hyperstructure multiplie les articles et surtout les angles d'attaque en exploitant des types de textes et des genres variés, avec des textes purement informatifs, d'autres à visée nettement explicative, d'autres à visée argumentative, parfois dans le cadre de controverses, etc. Ce faisant, alors qu'auparavant la question des responsabilités était souvent déniée ou diluée, elle devient un élément important du débat, en dépassant le cadre factuel du suicide et sa dimension émotionnelle. Les hyperstructures sélectionnées ci-dessous sont relativement emblématiques des représentations dominantes, sans qu'on décèle de distinctions nettes entre la PQN et la PQR, ce qui est en soi fort significatif de la façon homogène dont l'information est traitée.

On pourrait se demander ce qu'apporte l'hyperstructure par rapport à une analyse classique des événements. C'est l'occasion de préciser la nature des différences entre cette notion et les structures qui permettent de rendre compte des événements, tant chez Eliseo Veron ( 198I), Teun A. van Dijk (1985) que chez Francine Cicurel (1993). Ces auteurs insistent sur l'existence de structures d'intelligibilité, cognitives et

${ }^{10}$ Avec le format HTML (Hyper Text Markup Language). 
formelles, qui facilitent l'analyse de l'événement (en l'occurrence une catastrophe). Ainsi, autour de l'événement noyau, se greffe chez Eliseo Veron la prise en compte des conséquences, des événements antérieurs, la périodicité, l'arrière-plan, les réactions verbales et les histoires parallèles. Chez Teun A. van Dijk (1985), l'article combine commentaires (attentes, évaluations) et récit analytique de l'événement (événement principal, événements antérieurs, arrière-plan, circonstances, contexte, histoire). Ces matrices, qu'on retrouve chez Francine Cicurel (1993), opèrent au niveau du texte et ne concernent donc que la partie verbale de l'hyperstructure. Certes on les retrouve dans l'article central de l'hyperstructure, focalisée sur les composantes « centrales » de l'événement, mais aussi dans les autres modules textuels, susceptibles d'être consacrés à telle ou telle composante « périphérique » de l'événement. Plus fondamentalement, l'hyperstructure rompt avec l'existence d'un texte unique qui hiérarchise les données et les arguments, l'existence de modules plus ou moins autonomes favorisant des parcours de lecture plus diversifiés. Ce mode de présentation et d'analyse permet de rendre compte de la complexité en refusant tout point de vue ${ }^{\prime \prime}$ unique, posant en des termes inédits la question de la responsabilité journalistique. On y reviendra plus loin. Cet affaiblissement du texte primaire et unitaire implique que le journaliste fasse un effort particulier pour assurer une visibilité aux parties contractantes du pacte social afin qu'elles soient connues et reconnues (Honneth, 2000 ; Rabatel, 20 I0b).

\section{Les hyperstructures dans la presse quotidienne régionale}

Compte tenu de l'abondance des articles, on se centrera sur un exemple prototypique. Le Progrès (16/09/09), revient sur la question dans une hyperstructure qui couvre les trois quarts de la page $(6 \text { colonnes sur } 8)^{12}$ :

Image I : Le Progrès ( 6/09/09, p. 3)

\footnotetext{
"Qu'il s'agisse d'un point de vue énonciatif unique ou d'une perspective unique et unilatérale sur l'événement.

12 Merci à M.-L. Florea pour les schémas des hyperstructures.
} 
En haut de page, avec un titre sur 6 colonnes, un article (AI) confirme le tour formulaire des déclarations de Didier Lombard (PI) sur la contagion, en indiquant « Suicide et stress à France Télécom : la peur de la contagion. » Le titre de l'article articule, dans sa première partie, en position thématique « suicide » et « stress »: cette thématisation, qui correspond par définition à la reprise d'éléments co(n)textuellement saillants, signale que le lien entre le suicide et le stress est indiscutable - même si la nature sémantique du lien n'est pas précisée - comme le confirme le début de l'article. En effet, l'article, qui revient sur le $23^{e}$ suicide, inscrit le fait particulier non seulement dans une série, mais dans un cadre sociétal beaucoup général : « La pression au travail peut tuer. Ce n'est pas nouveau mais la vague de suicides et de tentatives chez France Télécom (23 depuis un an) met en lumière les dégâts causés par le stress au travail et les restructurations brutales chez les salariés » (Le Progrès, 16/09/09, p. 3).

Le Progrès rappelle également les mesures prises par Renault et PSA, qui ont eu à connaître de tels problèmes et ont tenté de les régler, tout comme EDF et la Société Générale - ce qui fait entendre par contraste, dans le contexte, le retard de France Télécom à prendre des mesures. L'article traite des responsabilités politiques, à travers le souhait de Xavier Darcos de faire entrer dans les faits l'accord interprofessionnel sur les conditions de travail signées en novembre 2008 entre patronat et syndicats, ainsi qu'à travers des déclarations de l'ANACT (Agence nationale pour l'amélioration des conditions de travail) qui confirment les résistances des décideurs (en évoquant un « tabou ») et soulignent l'importance d'agir alors que la crise pèse sur le moral des salariés.

L'hyperstructure comprend également un article statistique (A2) indiquant que « le suicide tue plus que la route », précisant qu'à France Télécom, « si l'on exclut les jeunes et les personnes de plus de 60 ans, le taux devient deux fois plus élevé que dans la population active située dans la même tranche d'âge ». Un troisième article (A3) donne la parole au délégué central de la CGC-UNSA (P2), syndicat qui a joué un rôle central dans la création de l'Observatoire du stress, qui évoque le « harcèlement institutionnalisé », la « hiérarchie de petits chefs » eux-mêmes soumis aux pressions de leurs supérieurs, citant le cas d'un salarié de « 52 ans, qui, en 5 ans, a changé cinq fois de métier au sein de l'entreprise. » L'article entrecroise très efficacement ce témoignage avec l'analyse de Dominique Decèze, un des premiers auteurs à s'être penché sur ces questions, à France Télécom, en 2004, avec La machine à broyer (réédité et augmenté en 2009). II rappelle que la baisse d'effectifs (de 160000 à 90000 salariés en 10 ans) ne s'est pas faite sans pression, malgré la phraséologie patronale des départs volontaires, avec notamment 22000 suppressions en trois ans pour le dernier plan qui concerne au premier chef les salariés de plus de 50 ans. II est intéressant de souligner la convergence de ces prises de positions, convergence encore accrue avec celle du sociologue Henri Vacquin (P3) dans l'interview qui suit (A4), dont les analyses sont confirmées par Dominique Decèze et, plus encore, par les témoignages de salariés apportés par Jean-Yves Claret. En effet, HenriVacquin confirme que « les 
suicides ont été occultés par les entreprises », que le cas de France Télécom n'est pas isolé, mais relève d'un

« management ultralibéral, fondé sur l'appât du gain et l'individualisation de la relation au travail. Actuellement, les organisations changent tous les deux mois, les structures aussi. Les mutations géographiques et professionnelles sont permanentes et sans accompagnement : c'est très vulnérabilisant pour les plus faibles. Certains sont parfois dans une solitude colossale [...] La multiplication des cellules d'écoute et des DRH ne sert à rien, tant que les conditions de déstabilisation au travail sont là. II faut réinterroger la relation à la hiérarchie et au travail » (Propos recueillis par Nathalie Mauret, Le Progrès ( 6/09/09, p. 3).

Enfin, un dernier article, intitulé « Malaise sur le web »(A5), rapporte des témoignages de salariés ${ }^{13}$.

Cette couverture de l'événement, large et variée, l'est davantage que cette seule hyperstructure remarquable le laisse penser, si l'on prend en compte le fait que l'ensemble de ces 5 articles fait suite, à trois jours de distance, à deux autres articles.

Image 2 : Le Progrès (13/09/09, p. 5).

Le 13 septembre 2009, un article (A I) du Progrès montre la photo de l'immeuble d'où s'était défenestrée une salariée (PI) et donne la parole à Xavier Darcos (P2) et à Didier Lombard (P3), tout en interviewant dans un deuxième article (A2) Marie Pezé (P4), experte auprès des tribunaux en psychopathologie du travail, responsable de la consultation Souffrance et Travail depuis 12 ans à l'hôpital MaxFourestier de Nanterre. Cette dernière souligne que « la violence du geste est

\footnotetext{
13 La lecture des articles sur l'internet permet d'accéder aux réactions des lecteurs. Ainsi l'article de C. Azzaro du Point (06/ I / /09) est-il suivi de témoignages de travailleurs de France Télécom particulièrement significatifs. Parfois, les prises de position sont tranchées, comme sur le site du Figaro, mais ce journal dont le lectorat est nettement à droite héberge des commentaires qui sont loin d'être homogènes et hostiles aux salariés de France Télécom, même si un certain nombre d'entre eux sont violemment anti-fonctionnaires.
} 
proportionnelle à la violence subie », formule qui est mise en valeur par un soustitre grasseyé et une taille de police supérieure au reste de l'interview. Marie Pezé refuse de psychologiser l'affaire, tout en mettant l'accent sur l'insuffisance de certaines mesures (suspension provisoire des mobilités ${ }^{14}$ ) ou sur la nocivité de décisions organisationnelles, comme :

« former des managers à la détection des signes de fragilité des salariés : ce sont les cadres qui donnent les ordres, prescrivent l'intensification du travail, les changements de poste. C'est donc à ceux qui infligent ce qui est insupportable qu'on va demander de détecter les signaux » (Le Progrès, 13/09/09, p. 5).

Bref, la mise en page donne toute sa place au caractère dramatique du dernier suicide sans céder au spectaculaire. L'analyse en contrepoint de Marie Pezé explique le suicide en en faisant le terme d'un processus désintégrateur ; qui plus est ; elle situe nettement les responsabilités en annonçant par avance d'autres suicides si des changements radicaux dans le management ne sont pas mis en œuvre.

\section{Les hyperstructures dans la presse quotidienne nationale}

La PQN consacre elle aussi des articles sous la forme d'hyperstructure aux drames de France Télécom, confirmant par là que le $23^{\text {e }}$ suicide marque une intensification dans le traitement médiatique de cette affaire dans l'ensemble de la presse écrite. Ainsi en est-il dans Libération.

Image 3 : Libération (14/09/09, p. 16).

L'ossature de la page est assurée par un article de Luc Peillon (AI), très pédagogique comme l'indique le titre, «Aux origines de l'épidémie de suicides ».

14 Jusqu'au 3 I octobre, précise Libération (|4/09/09). 
Les sous-titres prennent la forme de questions (« Combien de suicides à France Télécom ? », « Pourquoi une telle série ? », « Que font la direction et le gouvernement? ») auxquelles répond le corps de l'article, en s'appuyant sur des exemples très accusateurs : ainsi la jeune femme qui s'est défenestrée avait-elle vécu « une triple réorganisation. Après un changement de site il y a trois mois (de Courbevoie à Paris) un changement de structure (d'Orange à France Télécom), elle venait d'apprendre une réorganisation de son service. D'une manière générale, l'entreprise est en restructuration permanente depuis de nombreuses années » (Libération, 14/09/09, p. 16).

Les chiffres qui suivent (identiques à ceux cités dans Le Progrès du 16/09/09, et qu'on retrouve dans les dépêches d'agence) avec l'évocation des « démissions forcées », du programme «It's time to move », qui conduit une partie des cadres à devoir changer d'affectation tous les trois ans, accréditent aussi la thèse de la responsabilité de l'entreprise. Le rappel des mesures prises par la direction ou le gouvernement appelle donc des réserves identiques à celles qui se sont exprimées par des spécialistes interviewés dans les numéros des 13 et 16 septembre 2009 du Progrès, à cette différence très importante que dans Libération, la distance est directement prise en charge par le journaliste, qui ne passe pas par la médiation des experts ou des syndicalistes:

« Reste que l'agitation empathique du gouvernement ne doit pas faire illusion. Car c'est l'Etat qui est encore le principal actionnaire avec $26,65 \%$ du capital, et, surtout, l'employeur des 65000 fonctionnaires que compte encore l'ex-entreprise publique » (Libération, I4/09/09, p. 16).

Cette distanciation est également marquée par le dessin de François Ayroles (II), avec des salariés dans des cases/prisons, et une case vide. Libération complète l'article essentiel (AI) par une colonne de repères : sur le chiffre des suicides (A2), sur la structure du capital (I2), mettant en valeur la part majoritaire de l'Etat et donc sans doute, indirectement, sa responsabilité majoritaire. Suivent un portait de Jean-Denis Combrexelle (A3), directeur de Cabinet de Xavier Darcos, missionné par son ministre pour suivre ce dossier, et, enfin, l'évocation des mesures prises par Renault après les trois suicides du Technocentre de Guyancourt (A4). II se dégage de ces hyperstructures une identique volonté d'informer, d'expliquer, en facilitant un retour sur le passé et en recourrant à des experts, et, plus particulièrement, à des experts indépendants et clairement engagés, ce qui permet de prendre une position nette.

En témoigne encore l'hyperstructure de L'Humanité (21/09/09) : la « une » annonce qu'« on se suicide aussi au ministère » (de l'équipement), mentionnant, en surimpression sur la photo du ministère de l'écologie, un rapport interne du ministère sur le mal-être des agents de l'équipement. La « une » annonce enfin, dans un bandeau en bas de page, un grand entretien avec le psychiatre et psychanalyste Christophe Dejours à la page 4. Les pages 2 et 3 composent une vaste hyperstructure avec, en page 2, un article qui développe des informations relatives à l'épidémie de suicides cachée au ministère annoncée en une (AI). 
Cet article est accompagné, en son centre, d'une illustration reproduisant un visage déformé par une sorte de ressort remonté par une clé, comme si ce visage torturé - qui n'est pas sans évoquer les créatures déformées de François Bacon - exprimait on ne peut mieux la chronique d'un suicide annoncé. L'article, qui occupe l'essentiel de la page de gauche, se poursuit sur la première colonne de la belle page suivante. Un deuxième article (A2) recueille « le point de vue » d'un responsable de la CGT sur les répercussions sur la vie familiale qu'entraîne la surcharge du travail. En page 3, une brève (A3) précise le nombre d'agents en arrêt maladie pour dépression grave depuis un an (500), en contraste avec une illustration du ministère dans les locaux modernistes de la Grande Arche de la Défense (PI). En dessous, figurent un article (A4) avec des extraits du comité central d'hygiène et de sécurité sur la nécessité de « veiller à la promotion du bien-être au travail », puis un deuxième « point de vue » (A5) d'un secrétaire départemental de la CGT sur la surcharge de travail à la direction interdépartementale des routes du Nord. Enfin, l'éditorial, (A6), pointe sur la responsabilité de l'état actionnaire. Si cette hyperstructure se démarque des précédentes par la sureprésentation des élus de la CGT, elle ne s'en distingue pas dans la façon globale dont les responsabilités sont établies, tant en ce qui concerne l'entreprise que le système plus général de management.

Image 4 : L'Humanité (21/09/09, pp. 2-3).

La généralisation de l'emploi des hyperstructures pour traiter de ces suicides est un indicateur très fort du sens politique qui est donné, par les médias, pour penser la réduplication des suicides en véritable problème de société dont sont responsables au premier chef les entreprises. En effet, la multiplication des modules permet de donner la parole à d'autres acteurs (les salariés) ou à des experts qui confirment que les suicides, loin de se réduire à des fragilités personnelles, renvoient à un mode d'organisation de la production et à un type de relations en entreprise. Or, ces pratiques sont certes imputables à la direction de France Télécom mais elles sont aussi largement répandues. D'où l'importance 
d'un double mouvement, vertical, pour tenter d'établir les responsabilités dans l'entreprise, et horizontal, pour rappeler que, la question n'étant pas propre à une entreprise est un véritable problème de société qui a émergé au début des années $90^{15}$. Les modules des hyperstructures font ainsi écho à des analyses de chercheurs (Dejours, 2003 ; Linhart, 2004 ; Gaulejac, 2005, 2007 ; Dejours, Bègue, 2009) ou de journalistes spécialisés, tel Ivan du Roy, qui soulignent que les suicidés ou ceux qui font une tentative de suicide ont (ou avaient) en effet le plus souvent une longue expérience professionnelle derrière eux, étaient des salariés dynamiques et compétents, ayant mal réagi aux pratiques managériales déstructurantes et dévalorisantes qui les ont frappés ${ }^{16}$. Bref, le suicide émerge comme un révélateur de l'importance des pathologies psychosociales (boulimie au travail, insomnies, recours aux anxiolytiques, explosion des arrêts maladie, stress pouvant aller jusqu'à un épuisement nerveux - burn out) et des dysfonctionnements au travail (anonymat, manque de dialogue, rythme des restructurations, turn-over, concurrence entre salariés, mise au placard, licenciement ou harcèlement moral, absentéisme, baisse de productivité et de qualité, accidents du travail, démissions) ${ }^{17}$.

Du point de vue de la responsabilité énonciative, l'hyperstructure se présente comme une technique d'écriture grâce à laquelle les journalistes peuvent rendre compte de la complexité, tout en contournant la contrainte de simplicité, dans la mesure où chaque module est simple à lire et que l'absence de lien entre les modules laisse au lecteur la charge des mises en rapport. L'hyperstructure permet aussi de contourner la contrainte de la brièveté, puisque la somme des textes brefs ne produit pas le même effet qu'un texte unique de même longueur. Enfin, l'hyperstructure permet au journaliste de respecter la contrainte

\footnotetext{
${ }_{15}$ Les problèmes sont apparus parallèlement aux changements de politique managériale. Sur ce point, on lira avec profit le travail de M. Feynie. Ce dernier, consultant interne d'une entreprise publique (qu'il appelle LP), a rapporté pour son travail de thèse, en « ethnologue indigène », les évolutions qui, entre 1992 et 2002, ont touché LP lorsqu'elle a changé de statut pour pouvoir s'ouvrir à la concurrence. On se reportera notamment au récit de conventions internes aux cadres (Feynie, 2010 : 40-60), révélatrices de l'idéologie du management (ibid. : 64-66), aux analyses consacrées à la langue de bois managériale, idéalisante et ronflante, truffée de néologismes, de mots anglais, de métaphores guerrières, sportives ou cynégétiques (ibid. : 70-89), à la description des stratégies de masquage de la hiérarchie par l'établissement de relations plus égalitaires (à travers le tutoiement, l'usage des prénoms), mais qui restent néanmoins très dissymétriques, dans un climat de concurrence exacerbé par l'évaluation individuelle des performances, qui fait dire à un témoin : « Jamais on ne s'est autant tutoyé, embrassé, appelé par le prénom et jamais parallèlement on n'a dit autant de vacheries les uns sur les autres » (ibid. : 125). Sur la langue du management, voir également F. Cusin-Berche (2003).

${ }^{16}$ Ces suicides relèvent de ce qu'É. Durkheim nomme dans sa typologie le « suicide fataliste » de ceux qui se tuent parce qu'ils pensent que leur avenir est bouché et que leur vie est par trop oppressante. On est loin du « suicide altruiste » des sociétés traditionnelles ou du « suicide égoïste » des sociétés individualistes ; en revanche, est-on si loin que le prétend C. Lemieux (2010 : 98) du « suicide anomique » des sociétés en crise, dans la mesure où les valeurs et règles d'autrefois sont bafouées?

17 Voir I. du Roy (2009), C. Dejours, F. Bègue (2009) et A. Rabatel (20 I 0a).
} 
d'objectivité et de neutralité, contrainte certes discutable, comme les précédentes, mais qui est si forte qu'elle explique la tendance à la réduction du discours primaire et la propension à se retrancher des derrière des sources souvent mises en confrontation. Or, la multiplication des modules permet de donner la parole à une multitude d'acteurs différents, d'envisager des aspects divers et d'échapper au simplisme des confrontations systématiques (et attendues) qui se neutralisent. Par là, elle s'avère un instrument qui permet non seulement de penser la complexité, mais encore de faire une place à des fractions du corps social qui ne sont pas toujours « visibles » (salariés, experts) et de leur assurer ainsi une meilleure connaissance et reconnaissance (Honneth, 2000), tout en jouant sur des effets argumentatifs indirects très efficaces dès lors que la parole des acteurs est montrée, mise en image (fixe ou mobile), selon des procédés de convocation des discours inspirés des liens dans les hypertextes (Rabatel, 20 I0c). II est vrai que certains titres de presse tiennent ce discours complexe sans passer par la technique de l'hyperstructure, par exemple Politis du 17/09/09, L'Humanité du 21/09/09, Le Point du 06/ I / 09. Mais au fond, ces exceptions ne confirmentelles pas la tendance? Car elles concernent soit des titres engagés (quotidien L'Humanité - ou hebdomadaire - Politis) qui ne sont pas soumis aux mêmes contraintes de neutralité, soit un hebdomadaire d'« information » (Le Point) qui, du fait de son rythme de parution, n'est pas soumis aux mêmes contraintes de rapidité et de simplicité pour faciliter la captation du public. Sans compter qu'il faut intégrer l'évolution générale des attentes du public, à ces moments-là, critère qui explique la couverture que Le Point consacre à l'événement. On peut donc bien considérer que l'hyperstructure présente une technique scripturale particulièrement intéressante pour contourner les contraintes dans la presse quotidienne, technique qui suppose toutefois que le métier de journaliste ne soit plus exercé dans la solitude mais dans le cadre d'une rédaction qui prépare en amont ses dossiers afin de faciliter la couverture et l'analyse d'un événement complexe.

\section{Conclusion}

Nous conclurons sur quatre remarques ; les deux premières font le point sur la thématique du tabou et du déni des suicides et, notamment, des suicides au travail tandis que les deux dernières reviennent sur la portée des procédés sémio-linguistiques de la représentation médiatique des suicides au travail.

I. Si la mort est une réalité dont on peine à parler directement, le suicide, plus encore le suicide professionnel, est une de ces réalités sur lesquelles on a davantage de difficultés à mettre des $\operatorname{mots}^{18}$. Cette situation peut s'expliquer

18 Le télescopage entre mourir et tuer analysé par B. Gardin (1990, 2005b : 225) à propos d'un accident du travail («X est tué ») était déjà, dans un contexte très différent, le signe d'une difficulté qui ne peut que croître avec un suicide. 
par la prégnance des tabous de la mort. Car tabou il y a, contrairement à l'idée selon laquelle l'abondance des discours sur la mort dans les arts, les changements des pratiques (sida, soins palliatifs, euthanasie), des rituels mortuaires ou du traitement médiatique de la mort modifieraient la prégnance du tabou (Walter, 1994). En effet, outre que ces évolutions sont plus prégnantes dans le monde anglo-saxon - dans lequel le déni de la mort avait été particulièrement fort -, on est en droit de se demander si elles modifient en profondeur le tabou (Déchaux, 1996: 164), d'autant que ce dernier peut être renforcé par des dénis sociaux ou sociétaux. En témoigne l'argumentation emblématique de Laurent Zylberberg, directeur des relations sociales à France Télécom - en réponse à une question de la journaliste Isabelle Horlans sur le suicide d'un cadre quinze jours auparavant -, dans la mesure où la persistance du tabou de la mort par suicide, renvoyée au mystère des individus, est le socle qui permet ensuite de déployer le déni des responsabilités patronales:

« Vous comprendrez que je ne peux pas m'exprimer publiquement sur un cas individuel. Dans une société qui emploie près de 200000 personnes, dans le monde et environ 100000 en France, on est logiquement confronté à toutes sortes de situations. S'agissant de cette dame, je ne sais pas si son geste est lié aux conditions de travail, même si j'ai tendance à penser qu'il n'y a jamais une seule raison qui pousse les gens au suicide. Je ne crois pas en la mono causalité. [...] J'irai même plus loin : lorsqu'un de nos collaborateurs met fin à ses jours, peu importe qu'il mette ou non en cause sa hiérarchie ; dans tous les cas, je ne peux ni ne veux exclure, a priori, les conditions de travail comme une des raisons possibles de ce geste. Si quelqu'un accuse sa hiérarchie, l'important n'est pas que ce soit vrai ou non, mais qu'il le pense : cela veut dire qu'on a loupé quelque chose. C'est un drame pour sa famille, mais aussi pour l'entreprise » (France soir, 18/05/09).

Le directeur des relations sociales, qui ne veut pas s'exprimer sur un cas particulier, a néanmoins «tendance à penser »- il se fait même affirmatif («je ne crois pas ») - que la mono causalité du suicide doit être rejetée, affichant un relativisme lénifiant et vague (« on a loupé quelque chose ») qui exonère l'entreprise de l'examen de ses responsabilités. Comme si l'erreur de la direction était moins sa politique industrielle et son management que sa communication, amenant les salariés à penser ce qu'ils pensent, à tort. Bref, avec les suicides au travail se cumulent, d'une part, le tabou de la mort et plus spécifiquement du suicide, d'autre part, le déni de questions sociales vives, renvoyées à une histoire personnelle insaisissable et asocialisée.

Ces schèmes de pensée sont d'autant plus efficaces qu'ils sont largement partagés, car la plupart des acteurs directs et indirects sont dans le déni ou la difficulté de parler. À côté de la stratégie volontaire qui consiste à mettre sous le boisseau une vérité qui dérange ${ }^{19}$, les témoins et les proches peuvent se sentir coupables de n'avoir rien vu venir, ou d'avoir vu mais de n'avoir pas

19 M. Feynie (2010 : 107-1 16) montre combien il est difficile de pénétrer une entreprise, d'abord parce qu'il faut l'autorisation de l'employeur, ensuite parce qu'il est impératif de gagner la confiance des salariés. Lorsque ces conditions sont réalisées, l'enquêteur n'est pas tiré d'affaire pour autant, 
su empêcher l'issue fatale. La presse elle-même est peu armée pour traiter ces questions, et, de surcroit, doit compter avec des entreprises fournissant des supports publicitaires importants. D'où, initialement, une re-présentation du suicide limitée au déni des responsabilités managériales, à une approche psychologisante, avant de procéder à une première tentative d'explication qui oppose (et neutralise) les sources patronales et syndicales.

2. En dépit des avancées sur la reconnaissance du suicide parmi les psychopathologies professionnelles et des responsabilités de l'entreprise, il n'en demeure pas moins que les entreprises font de la résistance ${ }^{20}$ : ainsi contestent-elles leurs responsabilités devant la Caisse nationale d'assurance maladie. En effet, seulement un suicide sur 5 sur le lieu de travail - faisant automatiquement l'objet d'un signalement - est reconnu comme accident du travail. La responsabilité de l'entreprise est encore plus difficile à établir lorsque le suicide a lieu hors du lieu de travail ; néanmoins, un arrêt de la cour de cassation a étendu la jurisprudence dans un sens plus favorable aux salariés ${ }^{21}$. En outre, la remise en question des dérives de la logique entrepreneuriale et managériale libérale ne va pas jusqu'à remettre frontalement en cause le poids des actionnaires et de la financiarisation de l'économie. De plus, l'analyse reste encore sectorielle, privilégiant le secteur semi-public - ou, du moins, c'est ce type d'analyses portant sur ces genres d'entreprises qui a les faveurs des maisons d'édition -, alors que d'autres professions sont concernées : «Gardien de prison, policier, psychiatre, urgentiste ou pompier sont considérés comme des "métiers exposés" ou "métiers à risque". Des professions où l'on affronte la violence ou même la mort. Mais le sujet reste tabou » (France 5, La santé au travail, consulté le 25/09/09). Ce tabou du lien entre un milieu professionnel/milieu de vie pèse donc particulièrement lorsqu'il s'agit de secteurs de la fonction publique où la responsabilité de l'état dans les dérives managériales est directement engagée : ainsi un tabou et un déni persistants font-ils évoquer pudiquement un « malaise enseignant » de nature psychologique 22 . Sans bien sûr se borner à la fonction publique : car

car la publication du travail est souvent mal perçue tant par les responsables de l'entreprise que par les salariés de base ; dans les deux cas, M. Feynie évoque un sentiment de « trahison ».

20 À la date du 19 septembre 2010 et au cours des 15 jours qui ont précédé ce moment, 5 suicides étaient à noter, portant à 23 le nombre des salariés de France Télécom qui se sont donné la mort en 2010, à la date du 19/09/10 où nous bouclons ce texte.

21 Un arrêté de la cour de cassation (arrêt de la deuxième chambre civile du 22 février 2007) considère la tentative de suicide d'un salarié à son domicile comme un accident du travail, dans la mesure où le salarié était en arrêt maladie pour syndrome anxio-dépressif, ainsi que le faisait valoir la caisse primaire de sécurité sociale, contre l'avis de l'employeur (www .village.justice.com, « Le suicide lié au travail : qualification, causes, responsabilité de l'employeur », consulté le 25/09/09). L'évolution de la loi souligne que, même en l'absence de lettre, dès lors que des certificats médicaux ou des attestations établissent l'existence de troubles graves et concordants survenus par le fait du travail, la faute inexcusable contre l'employeur peut être retenue.

22 Sur les évolutions du management dans l'éducation nationale, l'enseignement supérieur et la recherche française, voir C. Laval (2009), I. et M. This Saint Jean (2009), ainsi que B. Cassin (2009). 
le tabou, comme le déni, est encore plus fort pour les suicides en milieu carcéral, pour les parias que sont « nos » prisonniers. Mais ici, l'interpellation éthique et politique dépasse le cadre de l'analyse des pratiques médiatiques.

3. L'amplification des suicides et de leur écho oblige la presse à changer ses modes de représentation des suicides avec la sériation, l'abandon du recours au seul montage de citations, la montée en puissance de l'analyse dans le discours primaire des journalistes (indépendamment des relais énonciatifs) ou dans une scénographie qui favorise la multiplication des angles d'analyse et des points de vue dans le cadre des hyperstructures. Ces procédés dépassent la confrontation patronat/syndicat, sans toutefois la supprimer, puisqu'en fin de compte la parole syndicale est légitimée. Ils permettent aussi de donner une analyse plus complète de la complexité de l'événement, parfois de façon commode, en évitant aux journalistes de faire des mises en relation, qui restent à charge des lecteurs, compte tenu de la raréfaction du discours primaire journalistique. Toutes ces évolutions témoignent que la nécessité de représenter cet irreprésentable qu'est la mort - et plus encore la mort au travail - incite les pratiques d'écriture journalistiques à se modifier. Fait notable, il ne s'agit pas de nier l'émotion ni le récit, il s'agit de les inclure dans une démarche explicative, argumentative, et d'envisager la question de l'action, selon une dialectique qui n'est pas sans rappeler celle par laquelle Jean-Marc Ferry (1991) pense les relations entre récit, interprétation, argumentation et reconstruction (Rabatel, Florea, 20l I), en un sens plus complexe que la simple technique du storytelling (Salmon, 2007).

4. L'évolution du traitement médiatique des suicides traverse toute la presse, tant la PQN que, fait notable, la PQR, les gratuits et les hebdomadaires. Cela témoigne que la presse peut, sous certaines conditions, jouer un rôle positif de médiateur social en ne réduisant pas l'espace social à deux groupes antagonistes qui se neutraliseraient; en ouvrant l'espace à d'autres acteurs ; en ne se contentant pas d'être un metteur en scène ou un porte voix; en dépassant le compte rendu événementiel pour tenter d'expliquer le cours des choses et d'aider à la réflexion et à la prise de décision. Nous avons si souvent interpellé la presse, au prisme de la responsabilité (Rabatel, ChauvinVileno, 2006a, 2006b ; Rabatel, Koren, 2008), qu'il est juste de souligner ici la dimension positive de la médiation sociale jouée par des médias d'information, ou, du moins, les potentialités de cette médiation, quand la presse s'en donne les moyens.

\section{Références}

Adam J.-M., 2005, Introduction à l'analyse textuelle des discours, Paris, A. Colin.

Adam J.-M., Lugrin G., 2000, «L'hyperstructure : un mode privilégié de présentation des événements scientifiques », Carnets du cediscor 6, pp. I33-| 49. 
- 2006, «Effacement énonciatif et diffraction co-textuelle de la prise en charge des énoncés dans les hyperstructures journalistiques », Semen 22, pp. 127- 144.

Arquembourg J., 2003, Le temps des événements médiatiques, Paris, De Boeck-INA.

Badiou A., 1988, L'être et l'événement, Paris, Éd. Le Seuil.

Baudelot C., Establet R., 2006, Suicide. L'envers de notre monde, Paris, Éd. Le Seuil.

Bonnafous S., Temmar M., éds., 2007, Analyse du discours et sciences humaines et sociales, Paris, Éd. Ophrys.

Broucker de J., 1995, Pratiques de l'information et écritures journalistiques. Paris, CFPJ.

Cassin B., 2009, « L'état schizophrène, Dieu et le nous raisonnable », pp. 35I -37I, in : Gori R., Cassin B., Laval C., dirs, L'appel des appels. Pour une insurrection des consciences, Paris, Éd. Mille et une nuits.

Cicurel F., 1993, «Pré-visibilité des discours journalistiques. À propos d'un événementcatastrophe », Carnets du Cediscor, I, pp. 55-76.

ClotY., 2008, « Le statut de la critique en psychologie du travail : une clinique de l'activité », Psychologie française, 53, pp. 173- 193.

Cusin-Berche F., 2003, Le « management » par les mots. Étude sociolinguistique de la néologie, Paris, Éd. L'Harmattan.

Déchaux J.-H., 1996, «Tony Walter. The revival of death », Revue française de sociologie, 37, pp. $|6|-164$.

Dejours C., 2003, L'évaluation du travail à l'épreuve du réel. Critique des fondements de l'évaluation, Paris, INRA Éd.

Dejours C., Bègue F., 2009, Suicide et travail, que faire? Paris, Presses universitaires de France.

Du Roy Y., 2009, Orange stressé. Le management par le stress à France Télécom, Paris, Éd. La Découverte.

Durkheim E., 1897, Le suicide, Paris, Presses universitaires de France, 1960.

Feynie M., 2010, Les maux du management. Chronique anthropologique d'une entreprise publique, Paris, Éd. Le Bord de l'eau.

Ferry J.-M., 1991, Les puissances de l'expérience. Essai sur l'identité contemporaine, Paris, Éd. du Cerf.

Gardin B., 1987, « Comment dire la mort d'un travailleurs? », Mots, I4, pp. I49- I69. Repris in Gardin B. 2005a, Paroles d'ouvrières et d'ouvriers. Textes édités et présentés par Gardin N. et Boutet J. Limoges, Lambert Lucas.

— 1990, « La sociolinguistique française », Lexikon der romanistischen Linguistik, I, pp. 224230. Repris in Gardin 2005b, Langage et luttes sociales. Textes édités et présentés par Gardin N. et François F. Limoges : Lambert Lucas.

Gaulejac de V., 2005, La société malade de la gestion. Paris, Éd. Le Seuil.

Gaulejac de V., Aubert N., 199I, Le coût de l'excellence. Paris, Éd. Le Seuil, 2007

Grosse E.-U., Seibold E., 1994, «Typologie des genres journalistiques », pp. 32-55, in : Grosse E.-U., Seibold, E., hrsg., Panorama de la presse parisienne, Frankfurt, Berlin, P. Lang, 1996. 
Guilhaumou J., 1998, La parole des sans. Les mouvements actuels à l'épreuve de la Révolution française, Fontenay aux Roses, ens Éd.

— 2006, Discours et événement, Besançon, Presses universitaires de Franche-Comté.

Herrenschmidt C., 2007, Les trois écritures. Langue, Nombre, Code, Paris, Gallimard.

Honneth A., 1992, La lutte pour la reconnaissance, trad. de l'allemand par P. Rusch, Paris, Éd. du Cerf, 2000.

Jankélévitch V., 1977, La Mort, Paris, Flammarion.

Krieg-Planque A., 2009, La notion de «formule » en analyse du discours, Besançon, Presses universitaires de Franche-Comté.

Langage et société, 2010, Linguistique légale et demande sociale: les linguistes au tribunal, 132.

Laval C., 2009, « La réforme managériale et sécuritaire de l'école », pp. 153-168, in : Gori R., Cassin B., Laval C., éds, L'appel des appels. Pour une insurrection des consciences, Paris, Éd. Mille et une nuits.

Lemieux C., 20 I0, «Vague de suicides aux usines Renault : relire Durkheim », pp. 97-99, in : Lemieux C., La sociologie sur le vif, Paris, Presses des Mines.

Libération, 2007, 20 ans de faits divers. Enquêtes sur les crimes qui ont secoué la France, Paris, Éd. Le Seuil.

Linhart D., 2004, La modernisation des entreprises, Paris, Éd. La Découverte.

Lugrin G., 2000, « Le mélange des genres dans l'hyperstructure », Semen, I3, pp. 65-96.

Mazière F., 2005, L'analyse du discours. Histoire et pratiques, Paris, Presses universitaires de France.

Moirand S., 2007, Les discours de la presse quotidienne. Observer, analyser, comprendre. Paris, Presses universitaires de France.

Mouriquand J., 1997, L'écriture journalistique. Paris, Presses universitaires de France.

Paveau M.-A., Rosier L., 2005, «Éléments pour une histoire de I'analyse du discours. Théories en conflit et ciment phraséologique ».Accès: http://www.johannes-angermueller. de/deutsch/ADFA/paveaurosier.pdf.

Politis, 2009, « Floués jusqu'au désespoir », 17 sept.

Questions de communication, 2009, « Journalistes et sociologues. Retour sur des luttes pour "écrire le social" », 16.

Rabatel A., 2006, «L'effacement de la figure de l'auteur dans la construction événementielle d'un journal de campagne électorale et la question de la responsabilité, en l'absence de récit primaire », Semen 22, pp. 7|-85.

- 2008, « Pour une conception éthique des débats politiques dans les médias : répondre de, devant, pour, ou les défis de la responsabilité collective », Questions de communication, 13, pp. 47-69.

— 2010a, « Le traitement médiatique des suicides à France Télécom de mai-juin à mi-août 2009 : la lente émergence de la responsabilité du management dans les suicides en lien avec le travail », Studia universitas Babeş-Bolyai, Philologia, Tome LV, vol. I, pp. 3 I-52. 
- 2010b, «Deux modes de représentation idéologique people du pouvoir, lors du premier anniversaire de la présidence de N. Sarkozy », Semen, 30, à paraître.

- 2010c, «Analyse pragma-énonciative des s/citations du site d'Arrêt sur images », Argumentation et analyse de discours, 4, Tel-Aviv, pp. I- I6. Accès : http://aad.revues.org/ index806.html.

- 2011, « La pluri-sémioticité des s/citations du site d'Arrêt sur images », pp. 13-36, in Jaubert A., Lopez-Munoz J.-M., Marnette S., Rosier L., et Stolz C. (eds), Citations 2. Citer pour quoi faire? Pragmatique de la citation. Louvain, Academia Bruylant.

Rabatel A., Chauvin-Vileno A., éds, 2006a, «Énonciation et responsabilité dans les médias », Semen, 22.

— 2006b, « La question de la responsabilité dans les médias », Semen 22, pp. 5-24.

Rabatel A., Florea M.-L., 201 I , «Re-présentations de la mort dans les médias d'information », Questions de communication, 19, pp. 7-18.

Rabatel A., Koren R., éds, 2008, « La responsabilité collective dans la presse », Questions de communication, 13, pp. 7-24.

Ricœur P., 1983, Temps et récit, Paris, Éd. Le Seuil.

Roy I. du, 2009, Orange stressée, Paris, Éd. La Découverte.

Salmon C., 2007, Storytelling, Paris, Éd. La Découverte.

Santé et travail, « Suicides, le travail en accusation », 60, 2007.

Schrepfer-André G., 2005, « Incidence des formes de reprise du SN régime des sP en selon x énonciatifs sur leur portée phrastique et textuelle », Langue française, I48, pp. 80-94.

This Saint Jean I.,This Saint Jean M., 2009, « Réforme ou assassinat de la Recherche et de l'enseignement supérieur? », pp. I69- 182, in : Gori R., Cassin B., Laval C., éds, L'appel des appels. Pour une insurrection des consciences, Paris, Éd. Mille et une nuits.

Van DijkT., 1985, « Discourse Analysis : Its Development and Application to the Structure of News », Journal of Communication, 33-2, pp. 20-43.

Veron E., 198I, Construire l'événement. Les médias et l'accident de Three Mile Island, Paris, Éd. de Minuit.

WalterT., 1994, The Revival of Death, London, Routledge. 